\title{
Crosslinking of Poly(vinyl chloride) Fibers with 2-Dibutylamino- 4,6-dimercapto-1,3,5-triazine in Water
}

\author{
Thomas JANDO and Kunio MORI* \\ Graboplast, Gyor H-9023, Hungary \\ *Department of Applied Chemistry, Faculty of Engineering, \\ Iwate University, Morioka 020, Japan
}

(Received December 4, 1989)

\begin{abstract}
Crosslinking of poly(vinyl chloride) (PVC) fibers has been studied by nucleophilic substitution with 2-dibutylamino-4,6-dimercapto-1,3,5-triazine (DB) in the presence of sodium hydroxide and tetra- $n$-butylammonium bromide (TBAB) in water. The effects of concentration of the different components, the temperature and the reaction time on the crosslinking of PVC fibers have been investigated individually to determine kinetic parameters and reaction mechanism. It has been found that the cationic surfactant (TBAB) has a significant effect on the formation of crosslinked polymer network. TBAB reacting with DB forms a mono-tetra- $n$-butylammonium salt of DB (DBTBA). This compound is born a crosslinking agent and surface active agent. To reach high crosslinking density, the concentration of DBTBA salt must be higher than the concentration of its saturation point. If the reaction temperature is lower than the $T_{\mathrm{g}}$ of PVC fibers the crosslinking density low (under $5 \%$ ) the reaction hardly occurs. The crosslinking reaction has been treated as a pseudo-first-order reaction. The rate constant $(k)$ is $0.0230 \mathrm{~min}^{-1}$ at $90^{\circ} \mathrm{C}$ and the activation energy is $55.5 \mathrm{~kJ} \mathrm{~mol}^{-1}$. Elemental analysis data prove the crosslinking reaction takes place. By decreasing the temperature of the reaction mixture, the ratio between crosslinking and branching decreases.
\end{abstract}

KEY WORDS Poly(vinyl chloride) Fiber / Crosslinking in Water / Dimercapto-triazine / Cationic Surfactant / Activation Energy / Nucleophilic Substitution/

It is widely known that poly(vinyl chloride) (PVC) fibers and cloth are weak to heat and solvents. The undesirable properties of PVC fibers have limited so far the use of PVC cloth products. The heat and solvent resistance of PVC can be improved by its crosslinking. Chemical crosslinking reactions have been carried out mainly in organic solvents ${ }^{1-3}$ or in melted state $e^{4-6}$ using nucleophilic agents. However, the crosslinking of PVC fibers and cloth have not been investigated yet because a crosslinked PVC is never spun to fibers and the way to crosslink PVC fibers under spinning has not been found yet.

On the other hand, chemical reactions in water also have been carried out to substitute the chlorine atom with nucleophilic agents. ${ }^{7-9}$
We reported that PVC particles can react with sodium hydrosulfide and sodium sulfide in the presence of swelling agents and onium salts in. water. ${ }^{10}$ This reaction in water suggests the possibility of crosslinking of PVC fibers and cloth in water, although, these sulfides induce the coloration of the fibers during the reaction and give low efficiency.

In this paper, to improve the heat and solvent resistance, the crosslinking of PVC fibers with 2-dibutylamino-4,6-dimercapto-1,3,5-triazine (DB) in the presence of onium salts and alkali in water is investigated on the crosslinking conditions, kinetic and mechanism. 


\section{EXPERIMENTAL}

\section{Materials}

PVC fibers, Teviron $7050\left(\bar{M}_{n}, 89.000\right.$; finess, $50 \mathrm{de} / 15 \mathrm{fil})$ were purchaced from Teijin Co., Ltd. DB was supplied by Sankyo Kasei Co., Ltd. Onium salts and alkalies were used in a reagent grade. Mono-tetra- $n$-butylammonium salt of DB (DBTBA) was synthetized. DB $(13.6 \mathrm{~g}), \mathrm{NaOH}(4.0 \mathrm{~g})$, and tetra- $n$-butylammonium bromide (TBAB) $(32.2 \mathrm{~g})$ were added to $200 \mathrm{ml}$ of water, and then stirred at $70^{\circ} \mathrm{C}$ for $60 \mathrm{~min}$. The reaction mixture was extracted with ether. The extract was cooled at $0^{\circ} \mathrm{C}$ to yield DBTBA crystals.

\section{Processing Procedures}

DB was added to an alkali aqueous solution, in order to form metal salt of DB in water. In the second step tetra- $n$-alkylammonium (TAA) salts were added to the solution. PVC fibers were immersed in the solution during the next step. The applied formulations and concentrations of components are shown in Table I. After the treatment of PVC fibers, the samples were washed in methanol at $20^{\circ} \mathrm{C}$ for $60 \mathrm{~min}$ and then dried at room temperature for 1 day.

\section{Testing Procedures}

The weight of the samples were measured before and after the treatment to determine the weight which increases during the reaction.

The equlibrium swelling ratio in gel and the gel content of the samples were measured in THF at $20^{\circ} \mathrm{C}$ for $48 \mathrm{~h}$ to determine the network chain density $\left(v_{\mathrm{e}}\right)$ from the Flory-Rehner ${ }^{11}$ equation. The nitrogen, carbon and hydrogen contents of the fibers were measured by $\mathrm{CHN}$ elemental analysis. To determine the sulphur content of the samples, the oxygen burning method was used. A JEOL JED-2000 energy dispersive $\mathrm{X}$-ray spectrometer was used to evaluate the sodium, bromide and sulphur contents of the specimens.

An IR spectrometer was used to determine the formation of the DBTBA salt. A FT-IR spectrometer was also used to identify the triazine-dimercapto units in the PVC fibers after the reaction.

\section{RESULTS AND DISCUSSION}

\section{Essential Components}

Materials used in this study can be divided into four groups according to their functions:

1. polymer,

2. crosslinking agent,

3. surface active agents,

4. acid acceptors.

PVC fibers are used as polymer. DB is the crosslinking agent having a very low $\mathrm{p} K_{\mathrm{a}_{1}}$ and being very acitive. TBAB is used mainly as surfactant. It works as a phase-transfer catalizer. Alkali acts as acid acceptor and activates $\mathrm{DB}$ by forming its salt. $\mathrm{NaOH}$ is used mainly.

Table I. The applied formulations to study the crosslinking reaction of PVC fibers

\begin{tabular}{lcccc}
\hline & & Concentration range & & Selected concentration \\
\cline { 3 - 4 } Components & Quantity range & Selected quantity & $\mathrm{mol} \mathrm{m}^{-3}$ \\
\cline { 5 - 5 } & & - & $100 \mathrm{ml}$ & - \\
Water & $100 \mathrm{ml}$ & $-16(\mathrm{VC}$ unit & $0.05 \mathrm{~g}$ & 8 (VC unit) \\
PVC fiber & $0.05-0.1 \mathrm{~g}$ & $0.4-184$ & $0.3 \mathrm{~g}$ & 11 \\
Onium salt & $0.01-5 \mathrm{~g}$ & $0-120$ & $0.71 \mathrm{~g}^{\mathrm{a}}$ & 22 \\
Alkali & $0-4 \mathrm{~g}^{\mathrm{a}}$ & $0-170$ & $0.088 \mathrm{~g}^{\mathrm{b}}$ & 22 \\
\hline
\end{tabular}

a In the case of TBAB.

b In the case of $\mathrm{NaOH}$. 
To crosslink PVC fibers in water three components DB, TBAB, and $\mathrm{NaOH}$ are essential ones. If one of DB or TBAB is absent from the aqueous solution, no crosslinking occurs at all. In the absence of $\mathrm{NaOH}$ the gel content decreases markedly.

Effect of Temperature, Time, and Types of Reagents

In Figure 1 the effect of crosslinking temperature on PVC gel content in the crosslinking of PVC fibers with DB, TBAB and $\mathrm{NaOH}$ in water is demonstrated. If the temperature of the solution is under the $T_{\mathrm{g}}$ of PVC, the reaction hardly occurs. In these conditions the gel content is always under $5 \%$.

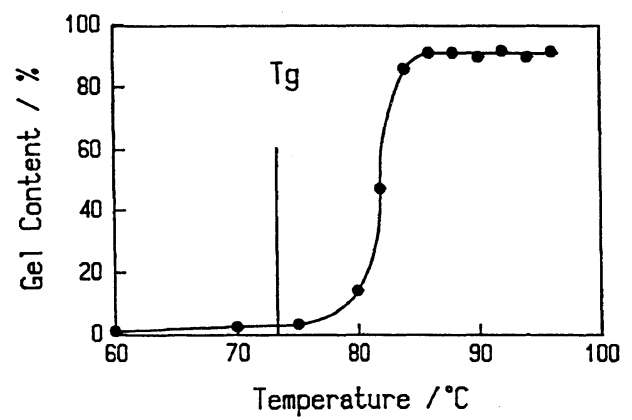

Figure 1. Effect of crosslinking temperature on the gel content of PVC fibers crosslinked with DB, TBAB, and $\mathrm{NaOH}$ in water for 60 min using the selected concentration show in Table I.

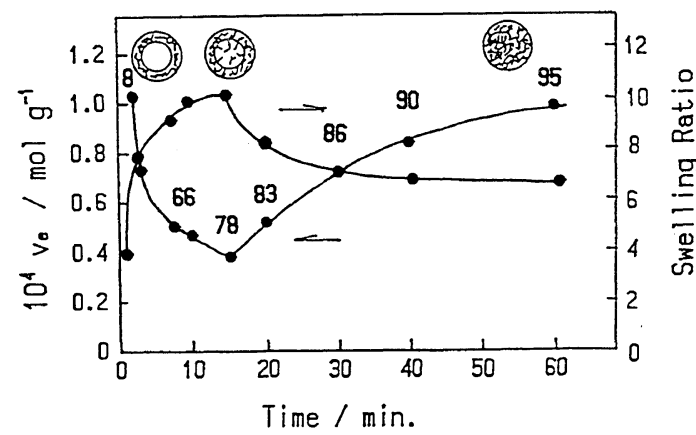

Figure 2. Effect of reaction time on the gel content, crosslinking density in gel $\left(v_{\mathrm{e}}\right)$ and swelling ratio of PVC fibers in the case of the selected concentration of DB, $\mathrm{TBAB}$, and $\mathrm{NaOH}$ at $96^{\circ} \mathrm{C}$, (numbers over the $v_{\mathrm{e}}$ curve indicate the gel content of PVC fibers).
If the temperature of the solution is higher than the $T_{\mathrm{g}}$ of the fibers, the gel content increases sharply and reaches its maximum value at $86^{\circ} \mathrm{C}$ for $60 \mathrm{~min}$. This shows that the segment movement of the polymer chains is a necessary condition for the crosslinking reaction to take place.

Next, the effect of crosslinking time on the gel content and crosslinking density in gel is investigated as shown in Figure 2. In increasing the reaction time, the gel content increased monotonously because the soluble ratio of the crosslinked fibers decreases monotonously. However, the crosslinking density in gel $\left(v_{\mathrm{e}}\right)$ decreased until a certain reaction time and then increased. To make this behavoir clear, we measured the swelling ratio of crosslinked PVC fibers as a function of time as shown in Figure 2. This is because the crosslinked polymer network has not been formed inside the PVC filaments yet. That is, the crosslinked polymer has been formed only on the surface of them during the first part of the reaction. Thus the swelling ratio is low and the calculated network chain density is high as Figure 2 shows. The minimum value of $v_{\mathrm{e}}$ and the maimum value of the swelling ratio indicate the formation of the crosslinked polymer network in the full cross-section of the filaments.

Figure 3 shows the effect of reaction time on the crosslinking density in gel at different

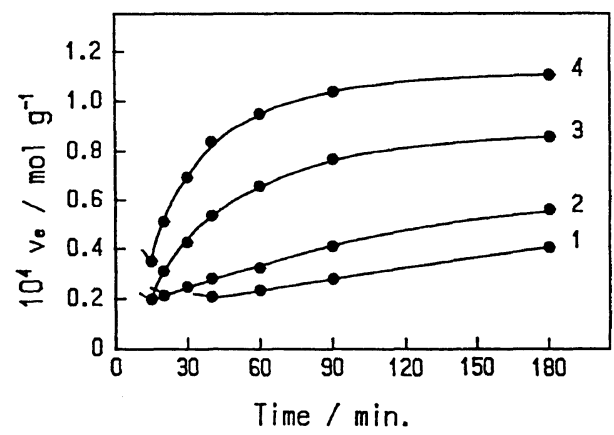

Figure 3. Effect of reaction time on the crosslinking density in gel $\left(v_{\mathrm{e}}\right)$ of PVC fibers in the case of the selected concentration of $\mathrm{DB}, \mathrm{TBAB}$, and $\mathrm{NaOH}$ at different temperatures: (1) $84^{\circ} \mathrm{C}$; (2) $88^{\circ} \mathrm{C}$; (3) $92^{\circ} \mathrm{C}$; (4) $96^{\circ} \mathrm{C}$. 
temperatures. The $v_{\mathrm{e}}$ decreases sharply and the time which belongs to the minimum value of $v_{\mathrm{e}}$ increases with the decreases of the reaction temperature.

Next, we studied the effect of various types of onium salts and alkalies on the reaction of PVC fibers with DB. In investigating the effect of onium salts on the weight increase as a function of reaction time at $96^{\circ} \mathrm{C}$, in the case of tetra- $n$-butylammonium chloride (TBAC), bromide (TBAB), and iodide (TBAI) the results do not show differeces. That is, the reaction rates are always the same. On the other hand, if we investigated the effect of tetra- $n$-butyl and tetra- $n$-octyl ammonium bromides (TBAB, TOAB), the rate of weight gain decreased with an increase in the length of alkyl chains (Figure 4). The mobility of the TAA salt of DB (DBTAA) decreases with the increase of carbon number of the alkyl chains. The $v_{\mathrm{e}}$ versus reaction time curves show similar differences than the weight increase versus reaction time curves. However, the gel content curves as a function of reaction time do not show these differences because the gel content changes sharply at the begining of the reaction and then reaches its maximum. It does not indicate the crosslinking process sensitively with the increase of reaction time.

Different types of alkalies $(\mathrm{KOH}, \mathrm{NaOH}$, $\mathrm{MgO}$ ) also do not change the rate of weight increase of PVC fibers according to our

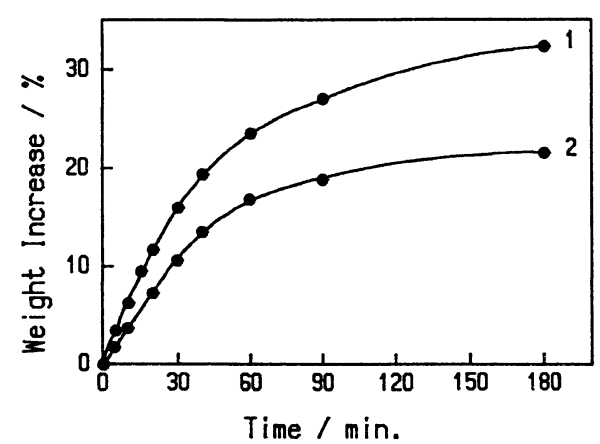

Figure 4. Time dependence of the weight increase of PVC fibers for TBAB (1) and TOAB (2) surface active agents in the case of the selected concentration of reagents at $96^{\circ} \mathrm{C}$. investigations.

\section{Concentration and Ratios}

The effect of the concentration of the crosslinking agent (DB), tetra- $\boldsymbol{n}$-alkylammonium salts and sodium hydroxide on the crosslinking has been investigated at $96^{\circ} \mathrm{C}$ according to Table I.

As Figure 5 shows with the increase of DB concentration, the gel content increase sharply until a critical DB concentration, in the case of different TBAB concentrations. After this critical concentration, the gel content remains constant as a function of DB concentration. The significant effect of the surface active agent can be observed very well. Without using TBAB, the gel content is under $5 \%$ even if the DB concentration is very high. Figure 5 also shows that the increase of gel content has a decreasing rate if the TBAB concentration rises.

The role of $\mathrm{NaOH}$ is illustrated by Figure 6. $\mathrm{NaOH}$ makes $\mathrm{DB}$ dissolved in water forming the sodium salt of DB (DBN) and reacts with the eliminating $\mathrm{HCl}$. It is not possible to reach the maximum gel content without using $\mathrm{NaOH}$.

The DB/TBAB ratio has an important role in the reaction. Curve No. 5 shows in Figure 7 , if the DB/TBAB ratio is higher than 1 , the gel content versus DB concentration curve rises slowly. If it is 1 or lower than 1 , the gel content increases sharply and reaches its maximum

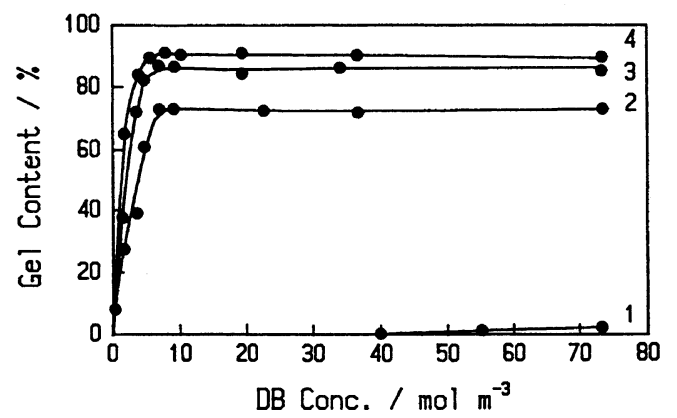

Figure 5. Effect of DB concentration on the gel content of the PVC fibers at different TBAB concentrations: (1) 0; (2) 4.7 ; (3) 15.5 ; (4) $46.5 \mathrm{~mol} \mathrm{~m}^{-3}$ at $96^{\circ} \mathrm{C}$ for $60 \mathrm{~min}$ (PVC concn $16 \mathrm{~mol} \mathrm{~m}^{-3} \mathrm{VC}$ unit, $\mathrm{DB} / \mathrm{NaOH}$ concn ratio $1 / 2$ ). 


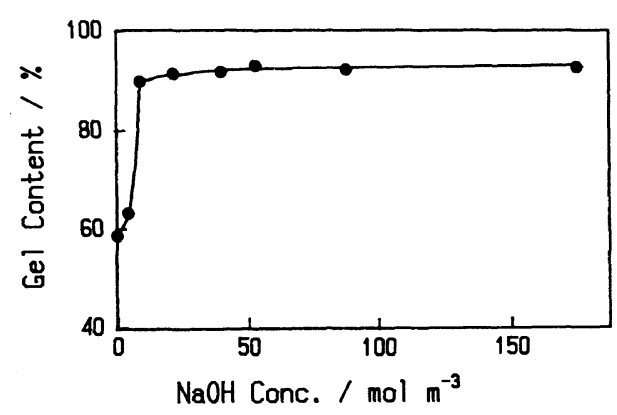

Figure 6. Effect of $\mathrm{NaOH}$ concentration on the gel content of PVC fibers at $96^{\circ} \mathrm{C}$ for $60 \mathrm{~min}$ (PVC concn $16 \mathrm{~mol} \mathrm{~m}^{-3} \mathrm{VC}$ unit, DB conc $11 \mathrm{~mol} \mathrm{~m}^{-3}$, TBAB concn $22 \mathrm{~mol} \mathrm{~m}^{-3}$ ).

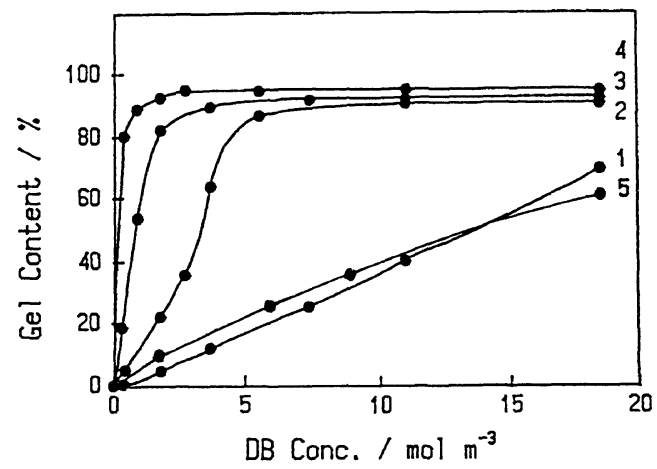

Figure 7. DB concentration dependence of the gel content of PVC fibers for different surfactants: (1) TPAB; (2) TBAB; (3) TPEAB; (4) TOAB at $1 / 2 \mathrm{DB} / \mathrm{TAAB}$ concentration ratio; (5) TBAB at $10 / 1$ DB/TBAB concentration ratio, at $96^{\circ} \mathrm{C}$ for $60 \mathrm{~min}$ (PVC concn $16 \mathrm{~mol} \mathrm{~m}^{-3} \mathrm{VC}$ unit, $\mathrm{DB} / \mathrm{NaOH}$ concn ratio $1 / 2$ ).

value at a critical reagent concentration as curve No. 2 indicates in Figure 7. These data demonstrate that if TBAB is in excess, compared to $\mathrm{DB}$, a higher degree of conversion can be reached at a lower crosslinking agent concentration. According to the above results we selected the crosslinking agent surfactant ratio $1 / 2$ for our experiments as can be seen in Table I. Crosslinking density and weight gain versus $\mathrm{DB}$ concentration curves at $1 / 2$ $\mathrm{DB} / \mathrm{TBAB}$ ratio have similar characteristics as gel content versus $\mathrm{DB}$ concentration curves at the same DB/TBAB ratio. They reach their maximum values at the same critical concentra- tion.

In investigating this critical concentration, we examined tetra- $n$-alkylammonium bromide (TAAB) surfactants with different alkyl chains (C3, C4, C5, C8), as tetra-n-propyl, butyl, pentyl, and octylammonium bromides (TPAB, TBAB, TPEAB, TOAB). The DB/TAAB ratio was $1 / 2$ at $96^{\circ} \mathrm{C}$ for $60 \mathrm{~min}$. It was found that in the increase for the number of carbon atoms of alkyl chains, the maximum gel content was reached at a lower DB concentration (Figure 7). According to our observations, the gel content increased sharply and reached its maximum value at that reagent concentration at which the DBTAA salt was saturated and precipitated. This is the so-called critical concentration. By increasing the number of carbon atoms in the alkyl chains, the solubility of the forming DBTAA salts in water decreases and the concentration of the saturation point decreases as well. The precipitated salt covers the surface of the PVC fibers and acts like a phase-transfer catalyst to carry the crosslinking agent to the fibers.

Separating and analysing the precipitated compound, using TBAB salt as surfactant, the IR spectra, elemental analysis and energy dispersive X-ray spectrometer data show the formation DBTBA salt. For example, in the IR spectrum of DBTBA the absorption peaks of the C-N stretching band at $1595 \mathrm{~cm}^{-1}$ and $\mathrm{C}-\mathrm{S}$ stretching band at $1130 \mathrm{~cm}^{-1}$, which show the presence of isocyanuric acid rings disappear because of the $S$-substituted DB salt formation. The results of elemental analysis of DBTBA salt (mp 79- $80^{\circ} \mathrm{C}$ ) are as follows: Calculated: N, $13.63 \%$; S, $12.48 \%$. Found: N, 13.2\%; S, $12.1 \%$. The energy dispersive $\mathrm{X}$-ray spectrometer data show that the salt does not contain $\mathrm{Na}$ and $\mathrm{Br}$ atoms.

The weight increase, crosslinking density in gel and sulphur content data of PVC fibers are summarized in Table II as a function of reaction time at $96^{\circ} \mathrm{C}$, to evaluate the effect of the forming DBTBA salt on the rate of crosslinking. As Table II shows, two kinds of 
Table II. Comparison of the weight increase, crosslinking density in gel $\left(v_{\mathrm{e}}\right)$ and sulphur content data for different compositions (I, II) in the case of the selected concentration at $96^{\circ} \mathrm{C}(\mathrm{I}, \mathrm{DB}, \mathrm{NaOH}, \mathrm{TBAB}$; II, DBTBA)

\begin{tabular}{|c|c|c|c|c|c|c|}
\hline \multirow[t]{3}{*}{ Time } & \multicolumn{2}{|c|}{ Weight increase } & \multirow{2}{*}{\multicolumn{2}{|c|}{$\begin{array}{c}\text { Crosslinking density } \\
10^{4} \mathrm{~mol} \mathrm{~g}^{-1}\end{array}$}} & \multirow{2}{*}{\multicolumn{2}{|c|}{$\begin{array}{c}\text { Sulphur content } \\
\%\end{array}$}} \\
\hline & \multicolumn{2}{|c|}{$\%$} & & & & \\
\hline & I & II & I & II & I & II \\
\hline 2 & 0.8 & 0.6 & 1.03 & 1.48 & & \\
\hline 4.5 & 2.6 & 2.7 & 0.609 & 0.454 & & \\
\hline 10 & 5.2 & 5.7 & 0.466 & 0.352 & & \\
\hline 15 & 9.0 & 9.4 & 0.352 & 0.336 & & \\
\hline 20 & 11.5 & 12.6 & 0.516 & 0.462 & 2.86 & 2.78 \\
\hline 30 & 16.0 & 16.4 & 0.690 & 0.670 & & \\
\hline 40 & 19.0 & 19.0 & 0.842 & 0.810 & 4.10 & 4.15 \\
\hline 60 & 22.0 & 22.3 & 0.947 & 0.910 & 4.92 & 4.87 \\
\hline 90 & 27.0 & 25.8 & 1.04 & 0.995 & 5.58 & 5.61 \\
\hline 180 & 32.3 & 33.4 & 1.12 & 1.07 & 6.13 & 6.27 \\
\hline
\end{tabular}

reaction mixture were examined. One of them contains $\mathrm{DB}, \mathrm{NaOH}$ and $\mathrm{TBAB}$ in the selected concentration, as Table I shows, and the other only DBTBA salt in the same concentration as DB in the previous composition. The results show the rates of reaction are the same in both reaction mixtures.

During the reaction of the sodium salt of DB and TBAB, the small sodium cation with its high charge density has a strong tendency to form $\mathrm{NaBr}$ salt with the bromide anion. In this way, between the large tetra- $n$-butylammonium cation and DB anion, an electrostatic interaction forming DBTBA salt takes shape. This salt has a double effect, acting as a surface active agent and as a crosslinking agent if its concentration reaches the saturation point. This is the reason why the DB/TBAB ratio must be maximum 1 or less; because, in this case, all of the crosslinking agents react with the surfactant and are activated by the applied surface active agent.

\section{Kinetics of Crosslinking Reaction}

The crosslinking reaction of PVC fibers in water can be regarded as a homogeneous reaction over the $T_{\mathrm{g}}$ of PVC fibers and over the melting point of DBTBA salt and of the reaction time which belongs to the minimum value $v_{\mathrm{e}}$, considerated as the induction period of the reaction, if the melted DBTBA salt covers the filaments. Thus the reagent and the fibers are in the same state and the DBTBA salt has diffused among the PVC chains. In this case the rate of crosslinking can be shown in eq 1

$$
v=k^{\prime}\left[-\mathrm{CH}_{2} \mathrm{CHCl}-\right]^{n}[\mathrm{DBTBA}]^{p}
$$

When $[\mathrm{DBTBA}] \gg\left[-\mathrm{CH}_{2} \mathrm{CHCl}-\right]$, as it was used during our experiments, eq 1 changes to eq 2

$$
v=k\left[-\mathrm{CH}_{2} \mathrm{CHCl}-\right]^{n}
$$

When eq 2 is converted in accordance with the crosslinking density versus reaction time curves, eq 3 and eq $4^{12}$ are given as follows

$$
\begin{gathered}
\ln \left[\left(\mathrm{d} v_{\mathrm{et}}\right)(\mathrm{d} t)^{-1}\right]=\mathrm{n} \ln \left(v_{\mathrm{emax}}-v_{\mathrm{et}}\right)+\ln k v_{\mathrm{emax}}{ }^{-1} \\
\ln \left[\left(v_{\mathrm{emax}}-v_{\mathrm{et}}\right) v_{\mathrm{et}}{ }^{-1}\right]=k\left(t-t_{\mathrm{o}}\right)
\end{gathered}
$$

where $v_{\text {emax }}$ and $v_{\text {et }}$ are the maximum value and the value at a $t$ time of the crosslinking density in gel respectively, $t_{0}$ is the reaction time at the minimum value of $v_{\mathrm{e}}$.

The kinetic parameters of the crosslinking 
Table III. Kinetic parameters of the crosslinking reaction of PVC fibers with DB, $\mathrm{TBAB}$, and $\mathrm{NaOH}$ in water in the case of the selected concentration

\begin{tabular}{|c|c|c|c|c|}
\hline \multirow{2}{*}{ Reaction order } & Temperature & Induction period & Rate constant & Activation energy \\
\hline & ${ }^{\circ} \mathrm{C}$ & $\min$ & $\min ^{-1}$ & $\mathrm{~kJ} \mathrm{~mol}^{-1}$ \\
\hline \multirow[t]{4}{*}{1.07} & 90 & 17.0 & 0.0230 & 55.5 \\
\hline & 92 & 15.0 & 0.0244 & \\
\hline & 94 & 14.0 & 0.0252 & \\
\hline & 96 & 13.5 & 0.0266 & \\
\hline
\end{tabular}

reaction were determined from Figure 3 . The reaction order $(n)$ was determined from the plot of $\ln \left[\left(\mathrm{d} v_{\mathrm{et}}(\mathrm{d} t)^{-1}\right]\right.$ versus $\ln \left(v_{\mathrm{emax}}-v_{\mathrm{et}}\right)$ in eq 3 and the rate constants $(k)$ where calculated from the plots of $\log _{10}\left(v_{\mathrm{emax}}-v_{\mathrm{et}}\right)$ versus $\left(t-t_{\mathrm{o}}\right)$ in eq 4. Our data are summarized in Table III. The reaction order is found to be a first order $(n=1.07)$ for an excess amount of DBTBA salt. If the DBTBA salt is not in excess the reaction order changes. Therefore the crosslinking of PVC is shown as a pseudo-first-order reaction. Similar results were reported by Mori ${ }^{13}$ in the case of the DB-MgO crosslinking of PVC in a melted state. The activation energy was

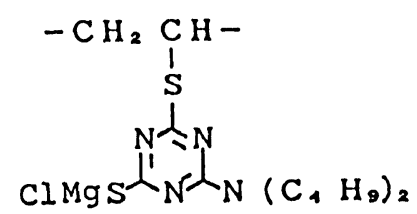

(I)

In the case of crosslinking of PVC fibers in the presence of $\mathrm{DB}, \mathrm{NaOH}$, and $\mathrm{TBAB}$ in water at $96^{\circ} \mathrm{C}, \mathrm{PVC}$ reacts with the forming DBTBA salt as shown in Table II. If we use such kinds of reagents which form DBTAA salt with the same structure, for example TBAC, TBAI, or $\mathrm{KOH}, \mathrm{MgO}$, the reaction rates do not alter; but if the structure of the DBTAA salt changes, as in the case of $T O A B$, the reaction rate also changes.

To understand the reaction mechanism, we calculated to be $55.5 \mathrm{~kJ} \mathrm{~mol}^{-1}$ for $k$. This value is significantly smaller than the activation energy of PVC dehydroclorination reaction, determined by Danforth ${ }^{14} 120 \mathrm{~kJ} \mathrm{~mol}^{-1}$.

\section{Mechanism of the Crosslinking Reaction}

It is known in the literature tht a DB-MgO system crosslinks the $\mathrm{PVC}$ at $180^{\circ} \mathrm{C}$. The VC units of PVC react with DBMg salt in two steps. ${ }^{13}$ The two mercapto groups of DB react one by one separately; in the first step, forming a branched substituted $\mathrm{VC}-\mathrm{DBMgCl}$ (I) structure and in the second step, the crosslinked VC-DB-VC (II) structure.

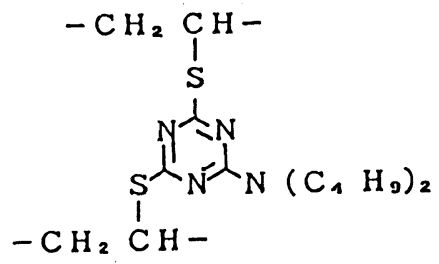

(II)

measured the nitrogen and sulphur contents of the treated PVC fibers by elemental analysis using different methods, and calculated from the weight increase of the fibers separately. In the case of calculation, we supposed that only substitution, forming branched structures III, IV, V) or only crosslinking (II) occurs separately. We set out from the following structures:

1. If only substitution occurs: VC-DBTBA (III), VC-DBN (IV), VC-DB (V) 
<smiles>CCSc1nc(N=[15NH])nc(SCC)n1</smiles>

(III)<smiles>CCSc1nc(N=[As])nc(SCC)n1</smiles>

(IV)<smiles>CCSc1nc(C)nc(S)n1</smiles>

(V)
2. If only crosslinking occurs: VC-DB-VC (II)

Nitrogen content versus weight increase curves show that the crosslinked polymer network is forming during the reaction (Figure 8). Curves No. 1, 2, 3, 4, agree with the above mentioned formulas II, III, IV, V, and show the calculated nitrogen contents from the weight increase if only branching (curves No. $1,2,3$ ) or only crosslinking (curve No. 4) takes place. Curve No. 5 indicates the measured nitrogen contents by elemental analysis reacting the PVC fibers at $96^{\circ} \mathrm{C}$. The measured nitrogen content data are higher than the calculated ones, if only branching occurs until a $22-26 \%$ weight gain. It is only possible if crosslinked polymer network (VC-DB-VC) forms during the reaction. In investigating the sulphur content data of PVC fibers as a function of weight increase similar results were given.

FT-IR spectrum of the treated specimens

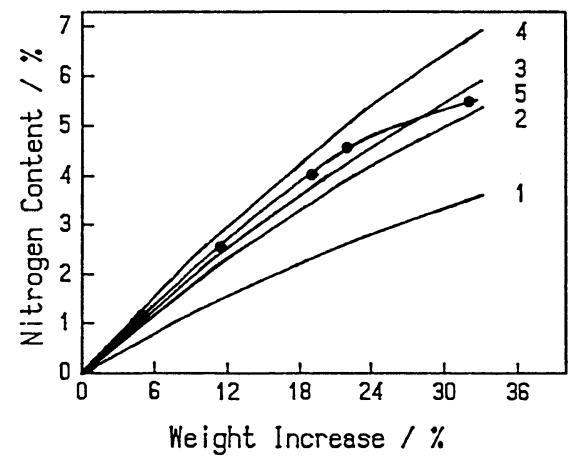

Figure 8. Calculated nitrogen content $(1,2,3,4)$ from the weight increase of treated PVC fibers and measured nitrogen content (5) of them as a function of weight increase in the case of the selected concentration of DB, TBAB, and $\mathrm{NaOH}$ at $96^{\circ} \mathrm{C}((1) \mathrm{VC}-\mathrm{DBTBA}$; (2) VC-DBN; (3) VC-DB; (4) VC-DB-VC). also demonstrate that the triazine-dimercapto units are built in among the PVC chains (Figure 9). The characteristic absorption bands of the triazine rings can be seen in the spectrum at $1560,1470,1380,1170$, and $860 \mathrm{~cm}^{-1}$ wavenumbers. The crosslinked structure can be estimated as the following formula VI and VII.<smiles>CSc1nc(N)nc(SC)n1</smiles>

(VI)<smiles>Cn1c(N)nc(=S)n(N)c1=S</smiles>

(VII)
The absorption bands at 1170 and $860 \mathrm{~cm}^{-1}$ suggest the formation of an $S$-substituted structure (VI). However the spectrum does not show the absorption bands based on the isocyanuric acid rings (VII) at 890 and $730 \mathrm{~cm}^{-1}$. During the crosslinking reaction $S$-substituted structure forms and $N$-substituted structure can not be detected.

In measuring the weight increase of PVC fibers as a function of reaction time at $96^{\circ} \mathrm{C}$

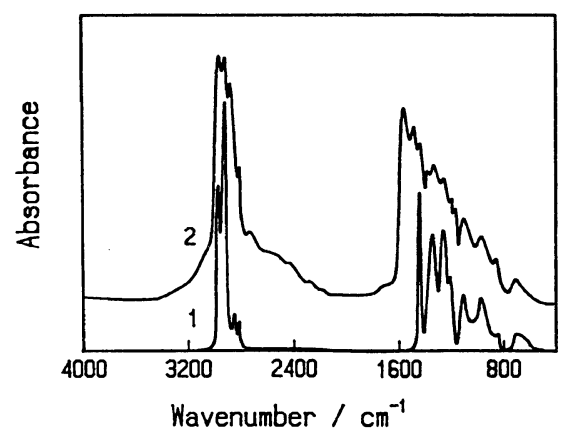

Figure 9. FT-IR spectra of uncrosslinked (1) and crosslinked (2) PVC fibers crosslinked at $96^{\circ} \mathrm{C}$ for $60 \mathrm{~min}$ in the case of the selected concentration of DB, TBAB, and $\mathrm{NaOH}$. 


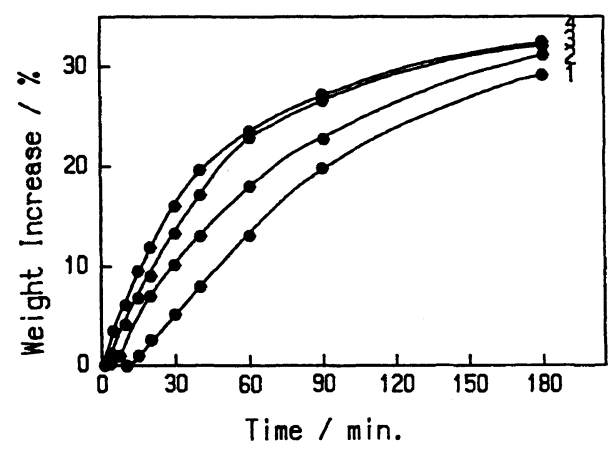

Figure 10. Effect of reaction time on the weight increase of PVC fibers at different temperatures: (1) $84^{\circ} \mathrm{C}$; (2) $88^{\circ} \mathrm{C}$; (3) $92^{\circ} \mathrm{C}$; (4) $96^{\circ} \mathrm{C}$; in the case of the selected concentration of DB, TBAB, and $\mathrm{NaOH}$.

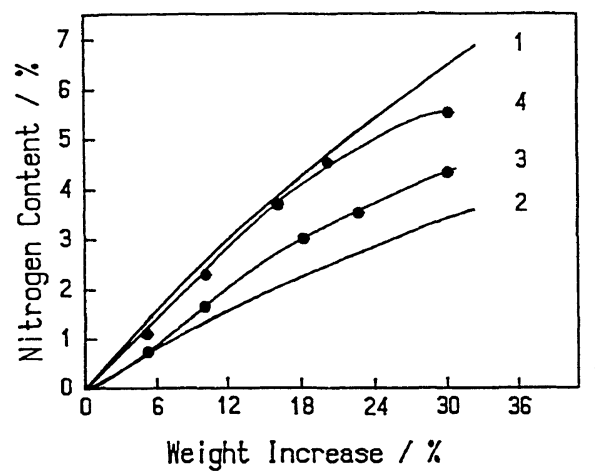

Figure 11. Effect of reaction temperature on the ratio of branching and crosslinking reactions of PVC fibers in the case of the selected concentration of DB, TBAB, and $\mathrm{NaOH}$ ((1) crosslinking (VC-DB-VC); (2) branching (VC-DBTBA); (3) $88^{\circ} \mathrm{C}$; (4) $96^{\circ} \mathrm{C}$ ).

(Figure 10), there are not so big differences among the curves measured at different temperatures than it was observed among the crosslinking density versus reaction time curves at different temperatures (Figure 3). These data suggest that with a decreasing reaction temperature, the reaction mechanism is changing; the ratio of substituted branched structures increases compared to the crosslinked ones. This is because with the increase of the ratio of branching, the weight of samples increases with a higher rate than with the increase of the ratio of crosslinking. To confirm this theory we measured the nitrogen content of PVC fibers at different temperatures. Nitrogen content versus weight increase curves are shown in Figure 11. Curves No. 4 and 3 indicate the measured nitrogen content of PVC fibers crosslinked at 96 and $88^{\circ} \mathrm{C}$. In decreasing the reaction temperature, the nitrogen content decreases, also. Curve No. 1 shows the calculated nitrogen content from the weight increase if only crosslinking (VC-DB-VC) occurs. Curve No. 2 shows the calculated nitrogen content from the weight increase if only branching (VC-DBTBA) occurs. Forming any kind of substituted, branched structures (III, IV, V) the nitrogen content of PVC fibers is lower than that in the case of crosslinking. These curves demonstrate that by increasing the reaction temperature the ratio of crosslinking increases.

The above mentioned data suggest that the crosslinking reaction of PVC fibers in water in the presence of DBTBA salt takes place in two steps. At first, one of the mercapto groups of DB reacts forming a branched structure and in the second step, crosslinking occurs. The values of ionization constants of DB back up this conception. The second ionization constant of $\mathrm{DB}\left(\mathrm{p} K_{\mathrm{a}_{2}}=11.4\right)$ is relatively high compared to the first one $\left(\mathrm{p} K_{\mathrm{a}_{1}}=4.1\right)$. This is the reason why mono-substituted salt of DB is forming. But after the reaction of DB salt with PVC, the ionization constant of the second thiol group decreases sharply $\left(\mathrm{p} K_{\mathrm{a}_{2}}{ }^{\prime}=4.8\right)^{15}$ and the crosslinking can take place.

According to our results the crosslinking reaction of PVC fibers in water in the presence of $\mathrm{NaOH}$ and TBAB salt takes place through the following mechanism. Mono-sodium salt of DB (DBN) forms adding DB to the water solution of sodium hydroxide (eq 1). The small sodium cation of DBN with its high charge density has a strong tendency to form $\mathrm{NaBr}$ salt with the bromide anion of TBAB. DB anion forms mono-tetra- $\boldsymbol{n}$-butylammonium salt with TBA cation (eq 2). The electrostatic interactions between the large tetraalkylammonium cation and DB anion are compar- 
atively weak, therefore the reactivity of $\mathrm{DB}$ anion is high. DBTBA salt reacts with a VC unit and the reactive DB anion substitutes the chlorine atom (eq 3). After this substitution the ionization constant of the second mercapto group decreases and its reactivity increases. Thus, the second mercapto group can react with another $\mathrm{NaOH}$ and $\mathrm{TBAB}$ molecule forming TBA salt again (eq 4). This branched PVC structure reacting with another VC unit forms the crosslinked structure (eq 5).

$$
\begin{gathered}
\mathrm{DB}+\mathrm{NaOH} \rightarrow \mathrm{DBN} \\
\mathrm{DBN}+\mathrm{TBAB}-\mathrm{DBTBA}+\mathrm{NaBr} \\
\mathrm{DBTBA}+\mathrm{VC}-\mathrm{VC}-\mathrm{DB}+\mathrm{TBAC} \\
\mathrm{VC}-\mathrm{DB}+\mathrm{NaOH}+\mathrm{TBAB} \\
-\mathrm{VC}-\mathrm{DBTBA}+\mathrm{NaBr} \\
\mathrm{VC}-\mathrm{DBTBA}+\mathrm{VC} \rightarrow \mathrm{VC}-\mathrm{DB}-\mathrm{VC}+\mathrm{TBAC}
\end{gathered}
$$

\section{CONCLUSION}

The reaction of PVC fibers with DB in water in the presence of surface active agents and alkalies was investigated with the following conclusions:

(1) The crosslinking reaction of PVC fibers in water takes place if the temperature of reaction mixture is higher than $T_{\mathrm{g}}$ of PVC.

(2) The crosslinked polymer network forms on the surface of PVC filaments at first.

(3) The surface active agent has a significant effect on the reaction of PVC fibers with DB. It forms a monotetraalkylammonium salt with DB. Therefore the ratio of the surface active agent and the crosslinking agent have to be at least $1 / 1$ in the reaction mixture.

(4) The forming DBTBA salt has double effect acting as a surface active agent and a crosslinking agent if its concentration is higher than the concentration of its saturation point.

(5) The crosslinking of PVC fibers with DBTBA salt is a pseudo-first-order reaction.
The activation energy was calculated to be $55.5 \mathrm{~kJ} \mathrm{~mol}^{-1}$.

(6) Nitrogen and sulphur content analysis and weight increase data verify that a crosslinking reaction takes place.

(7) FT-IR spectrum indicate the formation of an $S$-substituted structure.

(8) During the reaction, PVC reacts with the DBTAA salt forming $S$-substituted structure.

(9) The reaction takes place in two main steps. In the first steps, substituted branched structure and in the second step, the crosslinked structure form.

(10) By decreasing the reaction temperature the ratio of branching increases.

\section{REFERENCES}

1. M. Okawara and Y. Ochiai, in "Modification of Polymers," E. C. Carraher and M. Tsuda, Ed., ACS Symposium Series 121, Washington, DC, 1972, p 42.

2. Sankholkar, Macromol. Chem. Rapid Commun., 1, 613 (1980).

3. K. Mori and Y. Nakamura, J. Polym. Sci., Polym. Chem. Ed., 16, 2055 (1978).

4. V. Duhacek and A. Kuta, J. Appl. Polym. Sci., 27, 1549 (1982).

5. M. Behal and V. Duhacek, J. Appl. Polym. Sci., 37, 429 (1989).

6. G. K. Petrov and V. J. Prosvinkina, J. Prikladnoi Himii, 30, 1660 (1967).

7. M. Takeishi, Y. Naito, and M. Okawara, Angew. Macromol. Chem., 28, 111 (1973).

8. A. Gal, M. Cais, and D. H. Kohn, J. Polym. Sci., Polym. Chem. Ed., 16, 71 (1978).

9. R. O. Deanin and N. H. Ramolia, ACS Org. Coating Plastics Chem., 40, 193 (1979).

10. Y. Nakamura, K. Mori, and M. Saito, Kobunshi Ronbunshu, 36, 523 (1979).

11. P. J. Flory, J. Chem. Phys., 18, 108 (1950).

12. A. Y. Coran, Rubber Chem. Technol., 37, 673 (1964).

13. K. Mori and Y. Nakamura, J. Polym. Sci., Polym. Chem. Ed., 16, 1981 (1978).

14. J. D. Danforth, J. Phys. Chem., 87, 5376 (1983).

15. Kinki Chem. Ass., "PVC Basis and Application," Nikkan Kogyo Shinbun, Tokyo, 1988, p 156. 